\title{
Addendum: Highly inclined thin illumination enables clear single- molecule imaging in cells
}

Makio Tokunaga, Naoko Imamoto \& Kumiko Sakata-Sogawa

Nat. Methods 5, 159-161 (2008).

It has been brought to our attention that Konopka and Bednarek have independently developed a similar technique based on the use of highly oblique sub-critical incident angles in a microscope objective to create a tilted sheet of illumination that projects into a sample (Konopka, C. A. \& Bednarek, S. Y. Plant J. 53, 186-196; 2008). They used their technique, termed variable-angle epifluorescence microscopy (VAEM), for high-signal, low-background and near-real-time imaging of protein dynamics at or near the plasma membrane of plant epidermal cells and root hairs in whole, intact seedlings. Both VAEM and our highly inclined and laminated optical sheet (HILO) microscopy are based on the same concepts, but HILO microscopy changes not only the angle of the illuminating beam but also the radius and incorporates a field stop to reduce the divergence, resulting in higher signal/background ratios. Both techniques should be useful for imaging deeper into single or multicellular samples than is possible with total internal reflection fluorescence microscopy. 